\title{
An Investigation Into the Influence of Positive Peer Feedback on Self-Relevant Cognitions in Social Anxiety
}

\author{
Anne C. Miers \\ Unit Developmental and Educational Psychology, Institute of Psychology, Leiden University, Leiden, the Netherlands \\ Corresponding author: Anne C. Miers, Unit Developmental and Educational Psychology, Institute of Psychology, Pieter de la \\ Court Building, P.O. Box 9555, 2300 RB Leiden, the Netherlands. Email: acmiers@fsw.leidenuniv.nl
}

(Received 7 December 2020; accepted 17 May 2021; first published online 10 June 2021)

\begin{abstract}
This study investigated whether positive feedback from same-age peers can modify self-relevant cognitive processes of high socially anxious youth in a positive direction. Thirty-three high socially anxious and 32 non-socially anxious undergraduate students (17-22 years) gave an impromptu speech and received either positive or neutral feedback post-speech. Anticipatory processing (AP) was rated prior to the speech via self-report. One week later participants returned to the laboratory and completed questionnaires assessing post-event processing (PEP) thoughts related to the impromptu speech and AP about a future speech. For high socially anxious youth receiving positive feedback, AP about their speech performance significantly improved over time. In addition, high socially anxious participants who received positive feedback reported a higher frequency of positive PEP thoughts about their speech in the intervening week. These improvements did not occur in the neutral peer feedback condition. Non-socially anxious participants' AP improved in both feedback conditions, whereas their frequency of PEP was unaffected by feedback. These findings suggest that, in high socially anxious youth, positive feedback from same-age peers can modify selfrelevant cognitive processes in a positive direction. We discuss how methodological improvements could more thoroughly investigate the potential of positive peer feedback for changing cognitions.
\end{abstract}

Keywords: anticipatory processing; positive feedback; post-event processing; social anxiety; same-age peers

Social anxiety disorder (SAD) is a serious, chronic, and debilitating condition (American Psychiatric Association, 2013; Leigh \& Clark, 2018). It is defined by a marked and persistent fear of negative evaluation by others and avoidance of social situations (American Psychiatric Association, 2013). $\mathrm{SAD}$ is the most frequent anxiety disorder with a lifetime prevalence of approximately between 6 and 12\% (Knappe, Sasagawa, \& Creswell, 2015). Its personal, societal, and economic costs are high, and it is associated with increased risk for later depressive and substance use disorders, see Spence and Rapee (2016) for a review. SAD typically has its onset in early-to-mid adolescence; however, it is difficult to treat effectively in youth, with markedly lower remission rates for cognitive-behavioural therapy (CBT) than other anxiety disorders (Hudson et al., 2015) and therapy is often difficult to access. Moreover, subthreshold social anxiety symptoms are highly prevalent in adolescents and young adults that together with other factors (e.g., social functioning) place them at risk for developing SAD (Knappe et al., 2015; Spence \& Rapee, 2016). Interventions to help reduce social anxiety symptoms in individuals at-risk for developing SAD might benefit from targeting and emphasising cognitions related to the self, particularly positive self-cognitions (Gregory \& Peters, 2017; Thurston, Goldin, Heimberg, \& Gross, 2017). In this context, the current study investigates whether self-relevant

\footnotetext{
(c) The Author(s), 2021. Published by Cambridge University Press on behalf of the Australian Association for Cognitive and Behaviour Therapy. This is an Open Access article, distributed under the terms of the Creative Commons Attribution licence (http://creativecommons.org/licenses/by/4.0/), which permits unrestricted re-use, distribution, and reproduction in any medium, provided the original work is properly cited.
} 
cognitions in a sample of high socially anxious adolescents and young adults ${ }^{1}$ (aged 17-22 years) can be directly targeted and modified in a positive direction through the provision of positive feedback from same-age peers.

\section{Self-Relevant Cognitive Processes and Social Anxiety}

Research in both adult and adolescent populations indicates that individuals with high levels of social anxiety are caught in a cycle of negative self-relevant cognitive processes related to a social event (Leigh \& Clark, 2018; Norton \& Abbott, 2016). The prominent cognitive-behavioural models of SAD (Leigh \& Clark, 2018; Rapee \& Heimberg, 1997) identify self-relevant cognitive processes occurring before, during, and after a socially threatening situation as crucial to both the generation, and maintenance, of social anxiety. Before a social event, a socially anxious individual will engage in anticipatory processing (AP), expecting and predicting negative outcomes, such as poor social performance and social rejection (e.g., I think that new people I meet will think I am boring). During a social event, the individual will focus on his or her own negative feelings, bodily state information, and thoughts. After the social event, the individual will engage in post-event processing (PEP), described as a postmortem of the event. The individual reviews the event in detail, and, as generated during self-focused attention, negative thoughts and feelings predominate (e.g., I must have looked stupid). PEP can continue for days and weeks after the event and feeds into AP for future social situations. These self-relevant cognitive processes are theorised to exacerbate each other and therefore do not operate in isolation (Norton \& Abbott, 2016).

Recent review papers (Leigh \& Clark, 2018; Norton \& Abbott, 2016; Spence \& Rapee, 2016) describe correlational evidence consistent with the theoretical models that anticipatory and PEP maintain social anxiety symptoms during social events. Specifically, cross-sectional research shows that, compared with their non-socially anxious peers, youth with high levels of social anxiety show more negative AP before, and greater negative PEP after a social event (Leigh \& Clark, 2018; Spence \& Rapee, 2016). In one longitudinal study of 9-21 year-olds, negative PEP predicted an increase in behavioural avoidance over time, over and above the contribution of social anxiety (Miers, Blöte, Heyne, \& Westenberg, 2014). In a second longitudinal study, Blöte, Miers, Van den Bos, and Westenberg (2019) showed that shyness, measured at ages 9-16 years, was found to predict social anxiety symptoms 3-5 years later (after accounting for initial social anxiety levels) and this shyness-social anxiety link was mediated by negative self-relevant AP before a speech task (Blöte et al., 2019). Shy participants who had relatively negative perceptions of their own behaviour and performance during the speech at the start of the study were more likely to develop heightened social anxiety later. Stronger tests of maintenance come from experimental studies in (undergraduate) student samples, where experimentally heightened AP was shown to lead to higher levels of self-reported anxiety symptoms, see Norton and Abbott (2016) for a review. In a similar vein, PEP has been shown to maintain social anxiety via its sustaining influence on negative self-views in young adults (Norton \& Abbott, 2016).

\section{Positive Self-Relevant Cognitive Processes and Positive Peer Feedback}

There is growing evidence that intervention techniques might benefit from a stronger focus on enhancing positive affect and cognitions not only in the social anxiety (Asbrand et al., 2019; Goldin et al., 2013; Thurston et al., 2017) but also in the depression literature (e.g., Becker et al., 2019). Increasing positive self-thoughts are said to go a step further than only reducing negative self-thoughts, as such a reduction does not bring about a motivation to act and interact with the social world (Thurston et al., 2017). The suggestion is that positive self-views such as (they think I gave a good speech) would motivate a person to seek out new social experiences that are rewarding and generate positive affect.

\footnotetext{
${ }^{1}$ Henceforth, and in the interests of being concise we use the collective noun 'youth' to refer to our sample of adolescents and young adults.
} 
This view is supported by research showing that there are two different and independent biobehavioural systems, an avoidance system to prevent exposure to danger and an approach system to guide individuals towards potentially rewarding situations and that these systems are associated with distinct cognitive and affective processes (Kashdan, Weeks, \& Savostyanova, 2011). A review of positive psychological functioning in socially anxious individuals implies that a greater activation of the avoidant versus approach system is linked to dampened positive experiences (Kashdan et al., 2011). One way to address the under-activated approach system could lie in a greater focus on positive self-views. Positive self-thoughts are hypothesised to increase approach behaviour and motivation to engage with the social world (Thurston et al., 2017), hereby potentially activating the approach system and increasing the opportunity for reward. In this way, enhancing positive thinking should yield benefits that are otherwise out-of-reach when only negative thoughts are targeted.

A focus on enhancing the positivity of self-relevant cognitive processes is supported by research in adults showing that individual CBT for SAD increases positive self-views independently of decreases in negative self-views (Goldin et al., 2013). In addition, a study of early adolescents diagnosed with SAD showed an increase in positive PEP thoughts but not a decrease in negative PEP after standard group CBT versus a waitlist control group (Asbrand et al., 2019). Moreover, research in adult (Goldin et al., 2013; Thurston et al., 2017) and adolescent (Hogendoorn et al., 2014) samples indicates that increased positive (but not decreased negative) self-views are the mechanism driving the effect of CBT on (social) anxiety symptom reduction.

The aforementioned findings raise the question: if positive self-cognitions are an important mechanism, how then can these positive self-relevant cognitions be increased in high socially anxious youth? Current interventions such as cognitive-bias modification (CBM) largely focus on modifying negative cognitions to be less negative but do not explicitly target positive cognitions (Goldin et al., 2013; Reyes, Boulton, Han, Torok, \& Wong, 2020; Thurston et al., 2017). Positive and negative cognitions (e.g., automatic thoughts and interpretation bias) are shown to be relatively independent (e.g., the correlation between positive and negative automatic thoughts was -.38; Hogendoorn et al., 2010), and positive thoughts are shown to predict anxiety related to a speech performance independently of negative thoughts (Diaz, Glass, Arnkoff, \& Tanofsky-Kraff, 2001). This suggests that modifying a cognition to be less negative is not the same as modifying a cognition to be more positive. In the current study, we test an approach that directly targets the positive self-cognitions with the aim of increasing the positivity of high socially anxious youth's thinking.

This approach includes the social context within a brief intervention, specifically the use of sameage peers. Changes in the social environment during the second decade of life mean that same-age peers become highly motivationally salient (Steinberg \& Morris, 2001). With this changing social landscape comes a heightened sensitivity to both the positive and negative aspects of peer interactions (Kilford, Garrett, \& Blakemore, 2016). Peer interactions have intensified emotional salience, can be very rewarding, and provide unique opportunities for social learning (Haller, Kadosh, Scerif, \& Lau, 2015; Leigh \& Clark, 2018). Healthy young adults integrate positive, desirable social feedback from peers into their self-concept in a way that reinforces a positive self-image, and this feedback processing is associated with reward-related neural activity (Korn, Prehn, Park, Walter, \& Heekeren, 2012).

The potential for self-relevant cognitions of socially anxious individuals to be modified in a positive direction, via positive social feedback from same-age peers, is supported by a study in which participants' state self-esteem was found to be strongly contingent on social approval feedback from sameage peers (Reijntjes et al., 2011). In that study, early adolescents took part in a rigged online computer contest. They were told that they would be competing with four same-gender, same-age contestants from different schools; these contestants were fictitious. Furthermore, they were told that their personal profiles would be evaluated by 16 same-age peer judges. Participants were randomised to receive either approving, positive peer feedback or disapproving, negative feedback. Self-reported state selfesteem was assessed pre- and post-feedback. Findings showed that participants with elevated social anxiety experienced significant increases in state self-esteem following positive social feedback. Moreover, research indicates that adolescents with a tendency to experience anxiety have a heightened 
sensitivity to rewarding experiences with peers (Rosen et al., 2019). These studies are in accordance with the suggestion that socially anxious individuals' self-esteem is strongly contingent on other people's approval (Zou \& Abbott, 2012) and points to potential beneficial effects of positive peer evaluation for socially anxious youth.

\section{The Present Study}

The primary goal of this study was to test whether self-relevant cognitive processes in socially anxious youth can be modified in a positive direction, using positive peer feedback to directly target cognitions. In addition, we also tested the influence of positive peer feedback on self-relevant cognitions in a nonanxious group, as a comparison. Our youth sample is relevant because (1) during adolescence and young adulthood severe social anxiety symptoms manifest, placing up to a fifth of youth at-risk for developing SAD and (2) youth are susceptible to positive peer evaluations and these evaluations could, therefore, have a prominent influence on cognitive processes.

We investigated the influence of positive peer feedback on two different self-relevant cognitive processes: AP and PEP. Participants engaged in an impromptu speech task after which they received peer feedback. Prior to the speech task participants' performance expectations (i.e., AP) were assessed. One week following the task participants returned for a second session during which their thoughts about the speech during the previous week (i.e., PEP) were assessed as well as their performance expectations about a hypothetical future speech. Anticipatory cognitions relate to how well people think they will perform compared with age peers, scaled from performing less well (lower score) to performing (higher score). Modification of anticipatory cognition in a positive direction would entail an increase in AP score. As regards PEP, this cognition separately assesses the frequency of positive and negative thoughts about the speech; therefore, positive modification of this cognitive process would be evident in more frequent positive thoughts.

We chose to compare positive peer feedback to neutral feedback to have a stringent comparison as a control condition. We did not use negative feedback because abundant evidence has shown the detrimental effects of negative feedback (Koban et al., 2017; Reijntjes et al., 2011); hence, differences in self-cognitions between a positive and a negative condition cannot be unequivocally attributed to improvements as a result of positive feedback. Our goal was to create a neutral, or valence-free control condition. This is similar to control conditions used in CBM intervention studies (Reyes et al., 2020).

We hypothesised that positive versus neutral peer feedback would have a positive influence on high socially anxious youth, such that they would show more positive AP about the second (hypothetical) speech (hypothesis 1), and a higher frequency of positive PEP (hypothesis 2). In addition, in the context of the standard focus on modifying negative thinking processes, we also explored whether positive peer feedback would result in a lower frequency of negative PEP. As regards non-socially anxious youth, we did not make a specific prediction about the differential effect of feedback on self-relevant cognitions due to equivocal evidence. Drawing from the Zou and Abbott (2012) study, we would expect no difference between self-relevant cognitions in the positive and neutral condition. However, research also suggests that non-anxious individuals have a positively biased social learning about the self (Koban et al., 2017) suggesting that their self-relevant cognitions should benefit from positive peer feedback. Hence, the non-anxious group is not a standard 'control' group in the sense that intervention effects can be ruled out.

Prior to testing our main hypotheses and as would be expected from previous research (Leigh \& Clark, 2018; Miers, Blöte, Bokhorst, \& Westenberg, 2009), we checked that, pre-feedback, high socially anxious youth have significantly less positive AP about the speech task as compared with non-socially anxious youth. In addition, we verified that the impromptu speech task elicits heightened anxiety relative to the baseline and that high socially anxious report significantly higher state anxiety compared with their non-anxious counterparts (Anderson \& Hope, 2009). Finally, given recent evidence for the Bivalent Fear of Evaluation model (Weeks \& Howell, 2012) that describes how (some) socially anxious people fear positive (FPE) as well as negative evaluation, and its potential to obstruct the positive 
peer feedback effects, we controlled for FPE levels when testing the effect of positive versus neutral feedback on self-relevant cognitive processes.

\section{Method}

\section{Participants}

High socially anxious and non-anxious undergraduate students were recruited through an online university participant recruitment system, posters hung up around university buildings, and short presentations during first-year psychology lectures. Potential participants could respond to the advertisements about a study about "The science of online friendship" ${ }^{2}$ and sign up to the study through the university participation system. To ensure that persons with high levels of social anxiety would participate we also used advertisements that invited persons who are shy or who find it easier to chat with others online. Sixty-five first-year students $\left(M_{\text {age }}=19.42\right.$ years, $S D_{\text {age }}=1.66 ; 85 \%$ female $)$ participated for course credit. $47.7 \%$ of participants reported their origin as Dutch, $24.6 \%$ as non-Dutch European (the majority of which German), and the remaining $27.7 \%$ as other origins. In terms of the home situation, $46.2 \%$ of participants reported living with their biological mother and father and $53.8 \%$ had a different living situation. As both Dutch and non-Dutch students were invited to take part, the participants could choose using English or Dutch. $49.2 \%$ chose the English language, and 50.8\% chose Dutch. On the validated measure of social anxiety, the Social Anxiety Scale for Adolescents (SAS-A; La Greca \& Lopez, 1998), the high socially anxious group had a significantly higher social anxiety score $(M=55.06, S D=8.92)$ than the non-socially anxious group $(M=36.47, S D=5.80), t(63)=-9.93, p<.001)$. The minimum score in the high anxious group was 45 ; this is above the recommended cut-off score of 44 ( Olivares et al., 2002). The mean social anxiety scores per group are similar to previous studies with community youth samples (e.g., Miers et al., 2009). The anxiety groups did not differ on mean age, gender, language, nor home situation distribution (all $p s>.185$ ).

Four participants did not attend the second session. There was no difference on social anxiety level between those who attended both sessions $(M=46.15, S D=11.59)$ compared with those who attended the first session only $(M=42.25, S D=19.05 ; t(63)=-0.63, p=.533)$. The study was approved by the University's Psychology Department Ethical Committee. All participants provided written informed consent at the start of session one.

\section{Materials}

\section{Social anxiety}

The Social Anxiety Scale for Adolescents (La Greca \& Lopez, 1998) and its Dutch translation provided the measure of social anxiety. This 22-item instrument contains 18 descriptive self-statements about social anxiety symptoms (e.g., 'I worry about what others think of me' and 'I get nervous when I meet new people') and four filler items. Respondents are asked to rate each item according to the degree to which the item 'is true for you' $(1=$ not at all and $5=$ all the time). The total score ranges between 18 and 90. The SAS-A has good psychometric properties including in young adult samples (Blöte et al., 2019; Miers, Sumter, Clark, \& Leigh, 2020). Internal consistency as measured by Cronbach's $\alpha$ in the present study was good for both English, $\alpha=.95(n=32)$ and Dutch $\alpha=.91(n=33)$ participants.

\section{Anticipatory processing}

To measure participants' AP before the impromptu speech and in a hypothetical future speech, we used the Expected Performance measure (Spence, Donovan, \& Brechman-Toussaint, 1999) and its Dutch translation (Blöte et al., 2019; Miers et al., 2014). This 5-item questionnaire measures

\footnotetext{
${ }^{2}$ Participants gave a brief 'getting to know me' speech about themselves that is designed to reflect conversational speeches people have when first meeting someone to see if there is a match and a potential for friendship.
} 
participants' social self-cognitions in relation to giving a speech and their own performance, and fear about the upcoming speech. The latter item was not included in the calculation of the subscale score because it assesses state anxiety and not anticipated performance. An example item is 'Compared to other people your age, how well do you think you are going to do with this speech?' and 'How good do you think the judges watching your speech online will think you are at giving a speech?'. The items are rated on a 5 -point scale ( $1=$ lowest, $5=$ highest performance expectation). Internal consistencies for AP-before and AP-future were acceptable in both English, $\alpha=.78(n=32)$ and $\alpha=.80(n=29)$, and Dutch $\alpha=.71(n=31)$ and $\alpha=.78(n=31)$, respectively.

\section{Post-event processing}

Positive and negative PEP was measured with the Thoughts Questionnaire (TQ) and its Dutch translation (Miers et al., 2014). The questionnaire includes 14 items measuring negative thoughts (e.g., I must have looked stupid) and 9 items measuring positive thoughts (e.g., I came across as self-assured), and 1 neutral item. Two items per valence were added to assess thoughts about the feedback received post-speech, increasing the total number of items to 28 . These four items do not refer to thoughts about the self and are, therefore, not included in the positive and negative subscales. The items are answered on a 5 -point scale $(1=$ never to $5=$ very Often $)$ according to how often the participant had the thought in the week after the impromptu speech. One participant did not complete the PEP questionnaire. The internal consistency of the negative PEP subscale was $\alpha=.92$ in both English $(n=29)$ and Dutch $(n=31)$ participants, as was the positive PEP subscale $\alpha=.93$ (English, $n=29)$ and $\alpha=.90$ (Dutch, $n=31$ ).

\section{Feedback manipulation check}

Positivity of received feedback was rated by participants on a single-item measure with the question 'What do you think of the judges' feedback?' on a $10 \mathrm{~cm}$ visual analogue scale (VAS) ranging from not at all positive (0) to very positive (100).

\section{State anxiety}

To assess self-reported state anxiety, a VAS (Bond, Shine, \& Bruce, 1995) was used at several moments during session one. Participants rated their state anxiety level by answering the question 'How do you feel right now?' at different moments on a line of $100 \mathrm{~mm}$, ranging from not at all nervous (0) to very nervous (100). This functions as a proxy for state anxiety. Bond et al. (1995) validated this measurement tool: it highly correlates with the State version of the State-Trait Anxiety Inventory (i.e., STAI-S). Participants were instructed to mark their nervousness at five moments during the session: at baseline (VAS\#1), anticipatory nervousness rated after receiving speech instructions (VAS\#2), nervousness during the speech (VAS\#3), nervousness immediately after the speech (VAS\#4), and nervousness after receiving feedback (VAS\#5).

\section{Fear of positive evaluation}

Participants' fear of positive evaluation was assessed with the Fear of Positive Evaluation Scale (FPES; Weeks, Heimberg, \& Rodebaugh, 2008). This questionnaire includes 10 statements reflecting feelings of uneasiness associated with being evaluated favourably and in public. Example items are 'I am uncomfortable exhibiting my talents to others, even if I think my talents will impress them' and 'I generally feel uncomfortable when people give me compliments'. Statements are responded to on a 10-point scale according to the degree of agreement $(0=$ not at all true and $9=$ very true $)$. Two reverse worded items to detect response bias are excluded from the total score. The total score, therefore, ranges between 0 and 72. The FPES has demonstrated good psychometric properties (Weeks et al., 2008), and in the current study, the $\alpha$ was .83 for both English $(n=32)$ and Dutch $(n=33)$ participants. 


\section{Procedure}

\section{Overview}

Participants were told that the study was about the mechanisms behind online friendship building. The experiment consisted of two sessions both of which took place in the same university laboratory. During session one, the participants gave an impromptu speech of $3 \mathrm{~min}$ and received standardised positive or neutral feedback following the speech. The second session took place 1 week after the first session, to allow sufficient time for PEP (Edwards, Rapee, \& Franklin, 2003). The experiment was performed in a room that could be viewed from the outside through a one-way mirror. Instructions were provided by the experimenter via an intercom, or where appropriate in the procedure, face-to-face. Figure 1 depicts a flowchart of the study sessions and assessments.

\section{Feedback}

Participants were randomly assigned to either the positive or neutral feedback condition. Supplementary Table 1 shows baseline characteristics of participants in each condition. Positive and neutral feedback sentences were generated by a team of researchers and assessed in a pilot study in which undergraduate students $(n=55)$ rated several sentences on two aspects: valence and applicability to speech context. From these results, the positive and neutral feedback sentence sets were created. The feedback consisted of a total of eight sentences (four from each peer actor) that evaluated the participant in terms of the quality of the speech and the overall impression given by the person. For example, in the positive condition, a sentence about the speech was 'I think it was a good speech' and a sentence about the person was 'You come across as someone I could be friends with'. An example of a neutral sentence about the speech is 'Your speech was as good as others I've seen' and about the person is 'I think your hobbies are important to you'. To make the overall valence of the received feedback more realistic and appropriate within each feedback condition of eight sentences, two were of the opposite valence. Thus, in the positive condition, two sentences were neutral, one about the speech and one about the person and vice-versa in the neutral condition, two sentences were positive (one about speech and one about person).

\section{Session one}

After entrance to the lab, participants were asked to complete a number of instruments including the SAS-A and demographic questions on a laptop. Next, VAS\#1 was collected. Participants then sat at a small table in front of a PC screen. Next, two short films (each approximately $1 \mathrm{~min}$ ) were shown of a boy and a girl who were said to be earlier participants of the study (here termed 'peer actors'). In the films, the boy ('Lucas') and the girl ('Sara'), in this fixed order, gave an informal speech in which they spoke about themselves and their hobbies. After watching the videos, the participants were told that they have to give a speech lasting $3 \mathrm{~min}$ in which they introduce themselves and talk about two of their hobbies. Participants were told that the purpose of this 'getting to know me' speech was to allow the previous participants (Lucas and Sara) to decide if they would like to start an online friendship. We chose the subject's personal life and hobbies as the speech topic since in this way, there would be no between-subject differences in familiarity or knowledge concerning the topic.

Participants were told that they would be filmed and that the peer actors would be watching them via an online Skype connection while they were giving their speech. Furthermore, they were informed that after their speech, the peer actors would evaluate their speech and them as a person, so that mechanisms behind online friendship building could be studied. The participants were informed that this feedback would be given via Skype-chat. Before the experimenter walked into the room to set up the camera, the participant was asked to fill in the AP-before and VAS\#2 was assessed. After the experimenter set up the camera on its tripod, the participant received $3 \mathrm{~min}$ to prepare the speech and $3 \mathrm{~min}$ to perform the speech. During the preparation and speech phases, the participants were stood on a small, circular mat in front of the camera. Immediately after the speech participants first rated their state anxiety (VAS\#4) and then their anxiety during the speech (VAS\#3). After the 


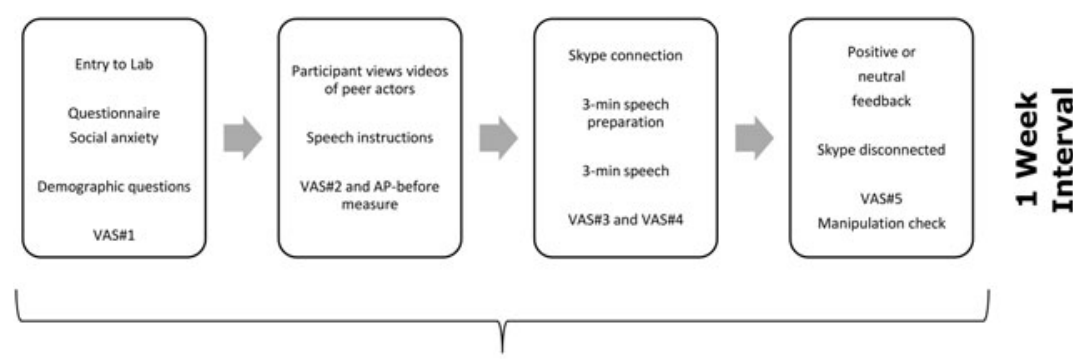

Session 1

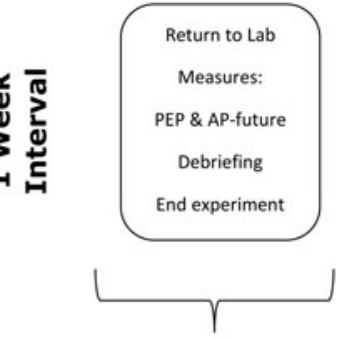

Session 2

Figure 1. Flowchart of experimental intervention. Abbreviations: AP = anticipatory processing; $P E P=$ post-event processing; VAS: visual analogue scale.

speech, the participant received standardised feedback (positive or neutral) via Skype-chat, and rated their state anxiety (VAS\#5) and positivity of the received feedback. The order of who gave the feedback first (i.e., Lucas or Sara) was randomised, as was the order of the sentences for each peer actor. At the end of the first session, the participant was told that in the next session, there would be an opportunity to chat with Lucas or Sara.

\section{Session two}

A week later the participant returned to the laboratory for the second session. First, the participant was asked to complete a few questionnaires, the TQ, and the AP-future, before they had the opportunity to chat with Lucas or Sara. After filling in these questionnaires, the participant was debriefed regarding the study goals and the deceptiveness of the feedback. During the debriefing, the experimenters gauged the degree of participant belief whether Lucas and Sara were real previous participants who gave real and valid feedback, and this was coded into the variable 'belief' $(0=$ showed belief and $1=$ expressed doubt). Participants who did not return for session 2 were debriefed via e-mail.

\section{Data Analyses}

\section{Preliminary analyses}

First, we conducted a manipulation check to test whether the positive feedback was rated by participants as more positive than the neutral feedback using an independent $t$-test. We also checked that the neutral feedback was not rated negatively, that is, that the neutral feedback was rated above 50 on the 0-100 VAS scale. Second, we checked that the impromptu speech task elicited increases in selfreported nervousness relative to baseline levels and conducted a between (social anxiety group) by within (time, five levels) mixed analysis of variance (ANOVA) on state nervousness. Third, we compared pre-feedback AP levels in anxiety groups with an independent $t$-test; AP-before was the dependent variable and social anxiety group the independent variable.

\section{Main analyses}

To test the first hypothesis (positive versus neutral peer feedback would have a positive influence on high socially anxious individuals, such that they would show more positive AP about the second (hypothetical) speech), we conducted a mixed between by within ANOVA, with time (before; future) as the within-subjects factor, feedback condition (positive and neutral) as the between subjects factor, and high socially anxious participants' AP-scores as the dependent variables. To test the second hypothesis (positive versus neutral peer feedback would have a positive influence on high socially anxious individuals, such that they would report a higher frequency of positive PEP), we conducted a multivariate ANOVA (MANOVA) with condition (positive; neutral) as the between subjects factor, and positive and negative thoughts as the dependent variables. These analyses were performed 
separately for the non-socially anxious group ${ }^{3}$. The sample size per anxiety group was sufficient to detect a between-within interaction with an effect size of partial $\eta^{2}=.25, \alpha=.05$, power $=0.80$. Achieved power to detect MANOVA effects were 0.69 for each anxiety group, medium effect size, $\alpha=.05$.

In the analyses for hypotheses 1 and 2, we added the variables FPE and belief in the cover story as covariates. When there were no significant main or interaction effects of the covariates, indicating that FPE, or belief did not influence the self-relevant cognitive process, analyses were repeated without the covariates and these findings are reported unless otherwise stated. Of the 248 total data points on the four dependent variables, four data points were missing $(0.016 \%)$, and these were missing completely at random (Little MCAR's test $\chi^{2}(6)=7.993, p=.239$ ) Before performing the main analyses, anticipatory and PEP were checked for normality assumptions for all group combinations. No outliers (scores greater than three standard deviations from the mean) were detected. Inspection of histograms and distribution tests indicated that the variables had distributions that approximated normality.

\section{Results}

\section{Preliminary Analyses}

First, an independent $t$-test showed that participants in the positive feedback condition rated the feedback as significantly more positive $(M=86.97, S D=10.35)$ than participants in the neutral condition $(M=72.59, S D=14.32), t(63)=4.65, p<.001$, Cohen's $d=1.15$. The same feedback difference was apparent in the high $\left(M_{\text {positive }}=86.75, S D=11.18 ; M_{\text {neutral }}=72.62, S D=13.87 ; t(31)=3.23, p=.003\right.$, Cohen's $d=1.13)$ and non-anxious $\left(M_{\text {positive }}=87.31, S D=9.36 ; \quad M_{\text {neutral }}=72.58, \quad S D=15.00\right.$; $t(29.83)=3.42, p=.002$, Cohen's $d=1.17)$ groups separately. The mean ratings of the neutral feedback indicate that it is not mistaken as negative feedback.

Second, as shown in Supplementary Figure 1, the procedure elicited a pattern of state anxiety commonly found in studies with impromptu speech tasks. A significant multivariate main effect for time was found, $F(4,59)=67.56, p<.001$, partial $\eta^{2}=.82^{4}$, indicating that state anxiety levels significantly changed over the course of session 1 . In addition, the main anxiety group effect was significant $F(1$, $62)=17.41, p<.001$, partial $\eta^{2}=.22$, indicating that, collapsed over all five anxiety measurements, high socially anxious participants reported significantly higher state anxiety than non-anxious participants. The interaction between time and anxiety group was not significant $(F(4,59)=1.52, p=.208)$. Pairwise comparisons collapsed across anxiety groups showed that baseline anxiety was significantly lower (at $p<.001)$ than all other VAS moments except post-feedback. Further, state anxiety levels at anticipation and during speech were significantly higher $($ at $p<.001)$ than state anxiety levels post-speech and post-feedback. Finally, post-speech anxiety was significantly higher than anxiety post-feedback $(p<.001)$.

Third, high socially anxious participants reported more negative AP pre-feedback as compared with non-socially anxious participants $\left(M_{\text {high }}=2.70, S D_{\text {high }}=0.55 ; M_{\text {non }}=3.09, S D_{\text {non }}=0.55 ; t(61)\right.$ $=2.82, p=.006$, Cohen's $d=0.70$ ). Whilst non-anxious participants expected to do at least as well as other people their own age in their impromptu speech, high anxious participants expected to give a poorer performance than other people their own age.

\section{Main Analyses: Positive Feedback Effects on Self-Relevant Cognitive Processes}

The analyses testing the first hypothesis (positive versus neutral peer feedback would have a positive influence on high socially anxious youth, such that they would show more positive AP about the

\footnotetext{
${ }^{3}$ We chose to perform separate analyses for the high and non-anxious group because we were primarily interested in the feedback effect on high socially anxious adolescents.

${ }^{4}$ Mauchly's test of sphericity was violated, $\mathrm{W}=0.59(9), p<.001$. The Greenhouse-Geiser correction test of within-subjects effect for time was significant, $F(3.11,192.55)=59.18, p<.001$, partial $\eta^{2}=.49$.
} 
second (hypothetical) speech) showed a main effect of time $F(1,27)=5.18, p=.031$, partial $\eta^{2}=.16$, indicating that, collapsed across conditions, AP significantly increased from session 1 to session 2. Although the time $\times$ condition effect was nonsignificant, $F(1,27),=1.51, p=.230$, the effect size was medium, partial $\eta^{2}=.05,95 \%$ CI $.000-.261$, suggesting that an effect is present but is not detected (type II error). We, therefore, conducted follow-up repeated measures ANOVAs per condition (with Bonferroni correction, $\alpha=.025$ ), with time (before, after) as the within-subjects factor ${ }^{5}$. The results of these analyses are displayed in Figure 2. In line with expectations, high socially anxious participants who received positive peer feedback showed a significant increase in their AP over time $\left(M_{\mathrm{AP}-\text { before }}=2.78, S D_{\mathrm{AP}-\text { before }}=0.56 ; M_{\mathrm{AP} \text {-after }}=3.13, S D_{\mathrm{AP} \text {-after }}=0.63, F(1,16)=6.19, p=.024\right.$, partial $\left.\eta^{2}=.28\right)$. In contrast, high socially anxious participants who received neutral feedback did not show an improvement over time nor did their expected performance ratings worsen $\left(M_{\mathrm{AP}-\text { before }}=2.58\right.$, $\left.S D_{\text {AP-before }}=0.59 ; M_{\text {AP-after }}=2.69, S D_{\text {AP-after }}=0.70, F(1,11)=0.66, p=.435\right)$.

The analyses among the non-socially anxious group testing the first hypothesis showed a main effect of time $F(1,28)=34.33, p<.001$, partial $\eta^{2}=.55$, indicating that, collapsed across conditions, AP significantly increased from session 1 to session 2 . Although the time $\times$ condition effect was nonsignificant, $F(1,28),=1.75, p=.197$, the effect size was medium, partial $\eta^{2}=.06,95 \%$ CI $.000-.266$. As with the high anxious group analyses, we therefore conducted follow-up repeated measures ANOVAs per condition, with time (before, after) as the within-subjects factor and Bonferroni corrected $(\alpha=.025)$. The results of these analyses are displayed in Figure 2. Non-socially anxious participants showed a significant increase in their AP over time in both the positive $\left(M_{\mathrm{AP}-\text { before }}=3.10\right.$, $S D_{\text {AP-before }}=0.62 ; M_{\text {AP-after }}=3.54, S D_{\text {AP-after }}=0.60, F(1,12)=34.13, p<.001$, partial $\left.\eta^{2}=.74\right)$ and neutral feedback $\left(M_{\mathrm{AP} \text {-before }}=3.03, S D_{\mathrm{AP} \text {-before }}=0.49 ; M_{\mathrm{AP} \text {-after }}=3.31, S D_{\mathrm{AP} \text {-after }}=0.38, F(1,16)=9.50\right.$, $p=.007$, partial $\left.\eta^{2}=.37\right)$.

Results of the analysis testing the second hypothesis (positive versus neutral peer feedback would have a positive influence on high socially anxious youth, such that they would report a higher frequency of positive PEP) are displayed in Figure 3. The multivariate main effect of condition was borderline significant $F(2,25)=3.52, p=.045$, partial $\eta^{2}=.22^{6}$. The follow-up univariate ANOVAs, controlling for belief and FPES, showed that high socially anxious participants in the positive feedback condition reported a significantly higher frequency of positive thoughts than high socially anxious participants in the neutral condition $\left(M_{\text {positive }}=2.16, S D_{\text {positive }}=0.60 ; M_{\text {neutral }}=1.68, S D_{\text {neutral }}=0.52\right.$, $F(1,26)=6.37, p=.018$, partial $\left.\eta^{2}=.20\right)$. There was no difference between high socially anxious participants receiving positive or neutral feedback in the frequency of negative thoughts $\left(M_{\text {positive }}=2.34\right.$, $\left.S D_{\text {positive }}=0.83 ; M_{\text {neutral }}=2.32, S D_{\text {neutral }}=0.94, F(1,26)=0.003, p=.959\right)$.

The MANOVA in non-socially anxious participants showed a significant multivariate effect of condition, $F(2,26)=3.78, p=.036$, partial $\eta^{2}=.23$; however, follow-up ANOVAs per condition were nonsignificant for both positive $(F(1,27)=3.68, p=.066)$ and negative $(F(1,27)=3.48, p=.073)$ PEP. $^{7}$

\section{Discussion}

The current study reported on a brief experimental intervention that employed positive feedback from peers about a speech task with the aim to directly modify two types of self-relevant cognitive processes typical of youth with high social anxiety: AP and PEP. We found that positive peer feedback significantly improved AP about a second (hypothetical) speech task in high socially anxious youth; neutral peer feedback did not change the level of AP. In addition, youth with high social anxiety who received positive feedback reported a greater frequency of positive PEP thoughts in the week post-speech, but they did not report a lower frequency of negative thoughts, as compared with youth who had received neutral

\footnotetext{
${ }^{5}$ See also https://statistics.laerd.com/spss-tutorials/mixed-anova-using-spss-statistics.php for comment on following up interaction terms.

${ }^{6}$ The covariate belief showed a significant multivariate effect $\left(F(2,25)=5.55, p=.010\right.$, partial $\left.\eta^{2}=.31\right)$, as did FPES $(F(2$, $25)=8.36, p=.002$, partial $\left.\eta^{2}=.40\right)$. Hence, the results reported use estimated marginal means.

${ }^{7}$ Hypotheses 1 and 2 were tested for females only. The same pattern of results was found, with similar effect sizes.
} 

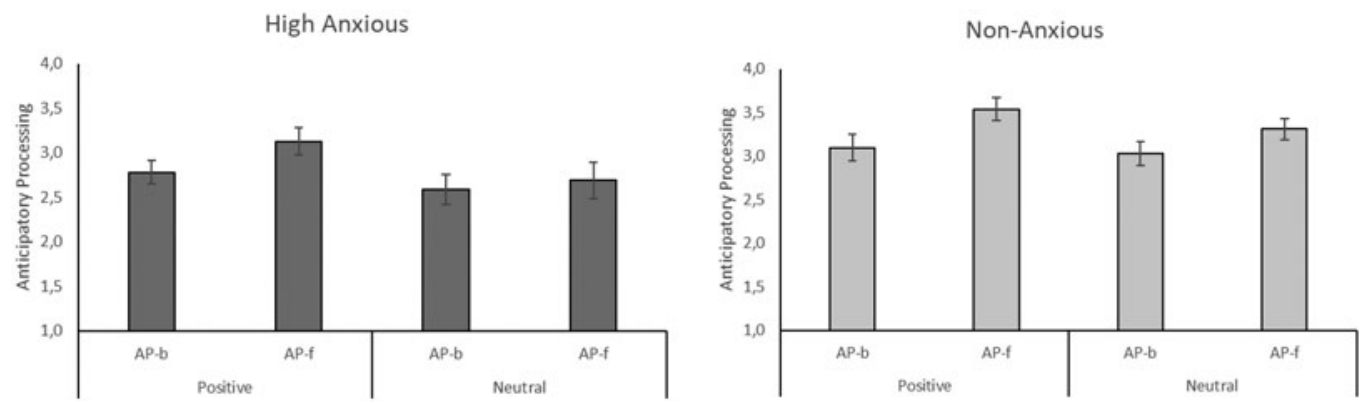

Figure 2. Speech AP by session and feedback condition, presented for high and non-socially anxious groups separately. Abbreviations: AP- $b=$ anticipatory processing before; AP- $f=$ anticipatory processing future speech. Note: For both anxiety groups, mean level of AP reported before the impromptu speech (AP-b) does not differ by condition ( $p s>.290)$.
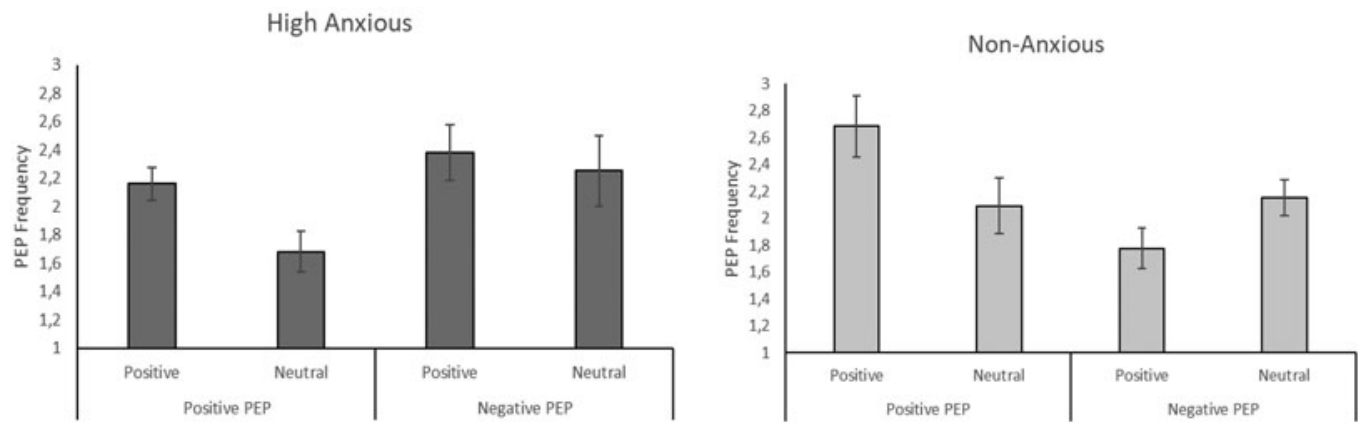

Figure 3. Positive and negative PEP by feedback condition, presented separately for high and non-socially anxious groups.

feedback. Non-socially anxious youth's AP increased in both feedback conditions; their PEP was unaffected by the feedback manipulation. As predicted, high socially anxious youth reported significantly more negative AP before the impromptu speech task as compared with non-socially anxious youth.

The positive influence of the positive peer feedback on self-relevant cognitive processes is in line with the study by Reijntjes et al. (2011). Their study included a younger sample, aged between 10 and 13 years, and measured the effect of positive peer approval within a computerised game on changes in state self-esteem before and after peer approval or disapproval. Reijntjes et al. (2011) reported a significant increase in state self-esteem in high socially anxious participants. Hence, as in the current study, socially anxious participants were responsive to peer approval, here in the form of positive feedback about a speech task. The current study extends the effect of positive peer evaluations not only to the theoretically pertinent cognitive processes specifically relevant to the self but also in terms of a maintained effect over the course of a week, not just an immediate improvement following peer approval.

The finding that in the positive compared with neutral peer feedback condition socially anxious youth had significantly more frequent positive thoughts about their speech is not entirely consistent with a study by Zou and Abbott (2012). In that study, differences between receiving a moderate versus a high score as feedback on an interaction task were found, but in a negative direction. That is, high socially anxious young people in the moderate condition reported significantly worse self-appraisal of their performance and higher negative rumination thoughts (PEP) immediately after receiving feedback. Positive rumination did not differ between the feedback groups. Stated differently, high socially anxious participants in the high score condition had better performance appraisal and fewer negative thoughts than participants in the moderate condition. Nevertheless, and taking the null finding for positive rumination into account, the feedback effects in the Zou and Abbott (2012) study are weighed more to the detrimental effects of 
moderate feedback as opposed to the beneficial effects of positive peer feedback. The divergent results could be explained by differences in feedback form and content (written vs. numeric in the Zou and Abbott (2012) study) or the source of feedback (same-aged peers vs. experimenter).

Negative PEP did not reduce nor worsen as a result of positive nor neutral feedback. Previous studies have shown reductions in negative self-views in adult samples (Goldin et al., 2013; Thurston et al., 2017), following 12 weeks of CBT. This implies that considerably more time might be required to adjust negative cognitive processes than the very brief 1 -week intervention presented here. Nevertheless, in a recent study with SAD youth aged 9-13 years, negative PEP did not reduce following 12 weeks of CBT, whereas positive PEP increased (Asbrand et al., 2019). It is possible that the selfrelated negative thoughts experienced by socially anxious youth following a social-evaluative situation, that is, PEP, are much less amenable to modification.

Finally, and consistent with a large body of research, we found that youth with high levels of social anxiety expect to perform worse than their age peers on a speech task; their AP is significantly more negative than the AP that non-socially anxious youth engage in (Leigh \& Clark, 2018; Miers et al., 2009). As shown in Supplementary Figure 1, this difference in expected performance was accompanied by a significant difference in self-reported anticipatory nervousness. Hence, in line with theoretical models of social anxiety, the prospect of an upcoming social-evaluative situation elicits negative AP and anxious feelings in individuals with social anxiety. These cognitions and emotions are experienced to a greater extent than in individuals with lower social anxiety.

Taking the potentially beneficial effects on self-relevant AP and PEP together, it appears that socially anxious youth can learn from the positive feedback delivered by same-age peers and help them to think in a way that their less anxious peers do. For example, socially anxious youth's scores on AP translate to an improvement from 'doing worse than others' pre-feedback to 'doing about the same as others', 1 week post-feedback (Figure 2). This post-feedback level is the same as the nonsocially anxious group prior to feedback. In a similar vein, the frequency of positive PEP in high socially anxious youth receiving positive feedback $(M=2.16)$ was as at least as frequent as in their nonanxious counterparts who received neutral feedback $(M=2.09)$. Such adjustments towards positive, and potentially more confident thoughts, whilst small in absolute terms, are interesting in the light of recent research highlighting the importance of enhancing positive self-views in therapeutic interventions for social anxiety (Goldin et al., 2013; Thurston et al., 2017).

The current study's focus on enhancing positive cognitions is in line with other recent intervention approaches that focus on positive dimensions, such as positivity-approach training for depressive symptoms (Becker et al., 2019) and Positive Affect Treatment (PAT) for depression and anxiety (Craske, Meuret, Ritz, Treanor, \& Dour, 2016). If, as suggested by Thurston et al. (2017), enhancement of positive self-thoughts would bring about behavioural engagement, activating the approach system (Kashdan et al., 2011) this could yield further benefits such as a reinforcement of positive responses in a young person's daily life and, in turn decreases social anxiety symptoms (Thurston et al., 2017). A next study using our approach could include the effects of positive peer feedback on more distal and consequential constructs such as self-esteem, social anxiety symptoms, and social approach behaviour.

Overall, the findings within the non-anxious group suggest that the feedback manipulation does not differentially influence their self-relevant cognitive processes. Regardless of feedback type, their AP increased from the first to second session and the frequency of positive and negative PEP did not differ between positive and neutral feedback conditions. The latter finding is in line with Zou and Abbott (2012) in which neither PEP nor performance self-appraisal measured up to $10 \mathrm{~min}$ post conversation improved in the positive versus moderate feedback condition. Whilst the finding concerning AP may be seen as contrary to that study, it does concur with research showing positively biased social learning in non-socially anxious individuals (Koban et al., 2017). The positive feedback likely served to reinforce the inherently positive self-views documented in healthy young adults (Rodman, Powers, \& Somerville, 2017). It can be speculated that the same occurred in the neutral condition but to a lesser extent via a focus on the few positive statements within the neutral feedback. Indeed, that the effect size for positive feedback (.74) was twice as large as for neutral feedback 
(.37), would seem to support this interpretation. Future research is required to replicate these effects and better understand why in non-socially anxious youth social learning from positive feedback seems not to extend to how they think about a social event after it has happened.

The current study has limitations. First, the analysis for the second hypothesis was underpowered; replication in a sufficiently powered study is, therefore, required using a screening procedure to select anxiety groups. Second, the sample consisted of young people with high levels of self-reported social anxiety and although the mean score was above the clinical cut-off on the anxiety scale, we cannot assume that the same positive feedback effects would be found in a group with a diagnosis of SAD. Third, the vast majority of the sample was female, and hence, we do not know the extent to which the positive feedback effects generalise to males. Given that available research does not show gender differences of positive feedback from peers on self-esteem (Reijntjes et al., 2011) and social learning (Koban et al., 2017) we would expect generalisation. Nonetheless, future studies should endeavour to recruit sufficient numbers of males and females to address this. Fourth, the design did not include a second social situation, such as a speech task or a social interaction. A future test of positive feedback could include a second speech task, post-feedback, to show that the influence transfers to an actual (rather than hypothetical) social situation. When faced with a second, anxiety-provoking speech task, negative self-views and beliefs may play a stronger role in the cognitive processes than when imagining the situation, thereby overriding potential positive feedback effects. Alternatively, as a result of habituation, a second speech task might not be as anxiety provoking as the first whereby the individual has the greater cognitive capacity to integrate the received positive feedback within their self-relevant cognitions. Moreover, within such a design additional cognitive and emotion variables could be included, such as the third self-relevant cognition featured in the cognitive models, self-focused attention, and selfreported state anticipatory anxiety to include a temporal match to the AP assessment. This would not only allow the investigation of improvements in self-relevant cognitive processes and state anxiety from one speech to another but also enable the investigation of the relations between cognitive processes and state anxiety, hereby providing a stronger test of the cognitive models. Related to this, the feedback effects were limited to self-report variables: future studies should include objective assessments of, for example, speech performance, by unknown peers to investigate potential behavioural improvements.

Future studies employing this brief intervention could also be improved by adding an assessment of positive feedback influence at a time longer than the 1-week interval studied here. This would show whether the temporal stability of positive feedback effects is maintained beyond this period of time. In addition, the measure of PEP was taken at only one time point, preventing the analysis of changes in this self-relevant cognition. Taking into account research showing fluctuations in the frequency of positive and negative PEP in the days following a social-evaluative task (Asbrand et al., 2019), we recommend assessing PEP thoughts on a daily basis, for example with Experience Sampling Methodology. Such an approach could be particularly useful for investigating the States-of-Mind ratio (SOM; Schwartz, 1997). According to the SOM model, psychological wellbeing is determined by a balance between positive and negative thoughts, rather than the absolute number of these thoughts (Schwartz, 1997). It would be interesting for future research to test whether positive peer feedback alters high socially anxious youth's SOM ratio such that it falls within an optimal range. Finally, improvements to this brief intervention should also focus on enhancing the believability that the received feedback was from same-age peers. Given that some participants expressed doubt about feedback validity, we cannot completely rule out an interpretation that receiving positive comments themselves improved self-cognitions, regardless of from whom the feedback was received. To be more confident about interpreting the results as showing the effect of feedback from peers specifically, the effect of receiving positive feedback from for example teachers versus same-age peers could be compared. Future studies could also use qualitative interviews with participants or funnel debriefing to probe their perception of the cover story and peer feedback and their ideas for making these methodological aspects more realistic, applicable, and believable.

In sum, the current study shows that positive peer feedback can be used to directly adjust selfrelevant cognitive processes in high socially anxious youth in a positive direction. Whilst recognising 
the need for replication in a larger, fully powered sample, with anxiety group selection using prescreening, these promising findings suggest that social approval from same-age peers may be a potential candidate for bringing about cognitive change. Provision of positive feedback in daily life could for example be provided through a mobile application that responds to a person's feelings about a social situation. In educational settings, attention could be given to ensuring that after a certain social activity, such as a presentation in the classroom, positive feedback from peers is included as well as from educational instructors. Future studies using this brief intervention are required to refine the procedure, investigate additional outcome measures, and examine its potential as a supplementary add-on intervention technique for socially anxious youth.

Supplementary material. The supplementary material for this article can be found at https://doi.org/10.1017/bec.2021.8.

Funding. This research received no specific grant from any funding agency, commercial or not-for-profit sectors.

Conflicts of interest. The author declares that she has no conflict of interest.

\section{References}

American Psychiatric Association (2013). Diagnostic and Statistical Manual of Mental Disorders (5th ed.). Washington, DC: American Psychiatric Association.

Anderson ER and Hope DA (2009). The relationship among social phobia, objective and perceived physiological reactivity, and anxiety sensitivity in an adolescent population. Journal of Anxiety Disorders, 23, 18-26. doi:10.1016/ j.janxdis.2008.03.011.

Asbrand J, Schmitz J, Krämer M, Nitschke K, Heinrichs N and Tuschen-Caffier B (2019). Effects of group-based CBT on post-event processing in children with social anxiety disorder following an experimental social stressor. Journal of Abnormal Child Psychology, 47, 1945-1956. doi:10.1007/s10802-019-00558-x.

Becker ES, Barth A, Smits JA, Beisel S, Lindenmeyer J and Rinck M (2019). Positivity-approach training for depressive symptoms: A randomized controlled trial. Journal of Affective Disorders, 245, 297-304. doi:10.1016/j.jad.2018.11.042.

Blöte AW, Miers AC, Van den Bos E and Westenberg PM (2019). Negative social self-cognitions: How shyness may lead to social anxiety. Journal of Applied Developmental Psychology, 63, 9-15. doi:10.1016/j.appdev.2019.05.003.

Bond AJ, Shine P and Bruce M (1995). Validation of visual analogue scales in anxiety. International Journal of Methods in Psychiatric Research, 5, 1-9.

Craske MG, Meuret AE, Ritz T, Treanor M and Dour HJ (2016). Treatment for anhedonia: A neuroscience driven approach. Depression and Anxiety, 33, 927-938. doi:10.1002/da.22490.

Diaz RJ, Glass CR, Arnkoff DB and Tanofsky-Kraff M (2001). Cognition, anxiety, and prediction of performance in 1st-year law students. Journal of Educational Psychology, 93, 420.

Edwards SL, Rapee RM and Franklin J (2003). Postevent rumination and recall bias for a social performance event in high and low socially anxious individuals. Cognitive Therapy and Research, 27, 603-617. doi:10.1023/a:1026395526858.

Goldin PR, Jazaieri H, Ziv M, Kraemer H, Heimberg RG and Gross JJ (2013). Changes in positive self-views mediate the effect of cognitive-behavioral therapy for social anxiety disorder. Clinical Psychological Science, 1, 301-310. doi:10.1177\% 2F2167702613476867.

Gregory B and Peters L (2017). Changes in the self during cognitive behavioural therapy for social anxiety disorder: A systematic review. Clinical Psychology Review, 52, 1-18. doi:10.1016/j.cpr.2016.11.008.

Haller SP, Kadosh KC, Scerif G and Lau JY (2015). Social anxiety disorder in adolescence: How developmental cognitive neuroscience findings may shape understanding and interventions for psychopathology. Developmental Cognitive Neuroscience, 13, 11-20. doi:10.1016/j.dcn.2015.02.002.

Hogendoorn SM, Wolters LH, Vervoort L, Prins PJ, Boer F, Kooij E and De Haan E (2010). Measuring negative and positive thoughts in children: An adaptation of the Children's Automatic Thoughts Scale (CATS). Cognitive Therapy and Research, 34, 467-478. doi:10.1007/s10608-010-9306-2.

Hogendoorn SM, Prins PJ, Boer F, Vervoort L, Wolters LH, Moorlag H, ... de Haan E (2014). Mediators of cognitive behavioral therapy for anxiety-disordered children and adolescents: Cognition, perceived control, and coping. Journal of Clinical Child \& Adolescent Psychology, 43, 486-500. doi:10.1080/15374416.2013.807736.

Hudson JL, Rapee RM, Lyneham HJ, McLellan LF, Wuthrich VM and Schniering CA (2015). Comparing outcomes for children with different anxiety disorders following cognitive behavioural therapy. Behaviour Research and Therapy, 72, 30-37. doi:10.1016/j.brat.2015.06.007.

Kashdan TB, Weeks JW and Savostyanova AA (2011). Whether, how, and when social anxiety shapes positive experiences and events: A self-regulatory framework and treatment implications. Clinical Psychology Review, 31, 786-799. doi:10.1016/ j.cpr.2011.03.012. 
Kilford EJ, Garrett E and Blakemore S-J (2016). The development of social cognition in adolescence: An integrated perspective. Neuroscience \& Biobehavioral Reviews, 70, 106-120. doi:10.1016/j.neubiorev.2016.08.016.

Knappe S, Sasagawa S and Creswell C (2015). Developmental epidemiology of social anxiety and social phobia in adolescents. In K Ranta, AM La Greca, L-J García-Lopez and M Marttunen (Eds.), Social anxiety and phobia in adolescents (pp. 39-70). Switzerland: Springer International Publishing.

Koban L, Schneider R, Ashar YK, Andrews-Hanna JR, Landy L, Moscovitch DA, ..., Arch JJ (2017). Social anxiety is characterized by biased learning about performance and the self. Emotion, 17, 1144. doi:10.1037/emo0000296.

Korn CW, Prehn K, Park SQ, Walter H and Heekeren HR (2012). Positively biased processing of self-relevant social feedback. Journal of Neuroscience, 32, 16832-16844. doi:10.1523/JNEUROSCI.3016-12.2012.

La Greca AM and Lopez N (1998). Social anxiety among adolescents: Linkages with peer relations and friendships. Journal of Abnormal Child Psychology, 26, 83-94. doi:10.1023/A:1022684520514.

Leigh E and Clark DM (2018). Understanding social anxiety disorder in adolescents and improving treatment outcomes: Applying the cognitive model of Clark and Wells (1995). Clinical Child and Family Psychology Review, 21, 388-414. doi:10.1007/s10567-018-0258-5.

Miers AC, Blöte AW, Bokhorst CL and Westenberg PM (2009). Negative self-evaluations and the relation to performance level in socially anxious children and adolescents. Behaviour Research and Therapy, 47, 1043-1049. doi:10.1016/ j.brat.2009.07.017.

Miers AC, Blöte AW, Heyne DA and Westenberg PM (2014). Developmental pathways of social avoidance across adolescence: The role of social anxiety and negative cognition. Journal of Anxiety Disorders, 28, 787-794. doi:10.1016/j.janxdis.2014.09.008.

Miers AC, Sumter SR, Clark DM and Leigh E (2020). Interpretation bias in online and offline social environments and associations with social anxiety, peer victimization, and avoidance behavior. Cognitive Therapy and Research, $\mathbf{4 4}$ 820-833. doi:10.1007/s10608-020-10097-1.

Norton AR and Abbott MJ (2016). Self-focused cognition in social anxiety: A review of the theoretical and empirical literature. Behaviour Change, 33, 44-64. doi:10.1017/bec.2016.2.

Olivares J, Garcia-Lopez LJ, Hidalgo MD, La Greca AM, Turner SM and Beidel DC (2002) A pilot study on normative data for two social anxiety measures: The social phobia and anxiety inventory and the social anxiety scale for adolescents. International Journal of Clinical Health Psychology, 2, 467-476.

Rapee RM and Heimberg RG (1997). A cognitive-behavioral model of anxiety in social phobia. Behaviour Research and Therapy, 35, 741-756. doi:10.1016/S0005-7967(97)00022-3.

Reijntjes A, Thomaes S, Boelen P, van der Schoot M, de Castro BO and Telch MJ (2011). Delighted when approved by others, to pieces when rejected: Children's social anxiety magnifies the linkage between self- and other-evaluations. Journal of Child Psychology and Psychiatry, 52, 774-781. doi:10.1111/j.1469-7610.2010.02325.x.

Reyes N, Boulton KA, Han J, Torok M and Wong QJ (2020). Cognitive bias modification for the induction of negative versus benign interpretations of the self in individuals with elevated social anxiety: Effects on self-related and anxiety outcomes. Cognitive Therapy and Research, 1-14. doi:10.1007/s10608-019-10074-3.

Rodman AM, Powers KE and Somerville LH (2017). Development of self-protective biases in response to social evaluative feedback. Proceedings of the National Academy of Sciences, 114, 13158-13163. doi:10.1073/pnas.1712398114.

Rosen D, Price RB, Ladouceur CD, Siegle GJ, Hutchinson E, Nelson EE, ..., Dahl RE (2019). Attention to peer feedback through the eyes of adolescents with a history of anxiety and healthy adolescents. Child Psychiatry \& Human Development, 50, 894-906. doi:10.1007/s10578-019-00891-8.

Schwartz RM (1997). Consider the simple screw: Cognitive science, quality improvement, and psychotherapy. Journal of Consulting and Clinical Psychology, 65, 970. doi:10.1037/0022-006X.65.6.970.

Spence SH and Rapee RM (2016). The etiology of social anxiety disorder: An evidence-based model. Behaviour Research and Therapy, 86, 50-67. doi:10.1016/j.brat.2016.06.007.

Spence SH, Donovan C and Brechman-Toussaint M (1999) Social skills, social outcomes, and cognitive features of childhood social phobia. Journal of Abnormal Psychology, 108, 211-221. doi:10.1037//0021-843x.108.2.211.

Steinberg L and Morris AS (2001). Adolescent development. Annual Review of Psychology, 52, 83-110. doi:10.1146/ annurev.psych.52.1.83.

Thurston MD, Goldin P, Heimberg R and Gross JJ (2017). Self-views in social anxiety disorder: The impact of CBT versus MBSR. Journal of Anxiety Disorders, 47, 83-90. doi:10.1016/j.janxdis.2017.01.001.

Weeks JW and Howell AN (2012). The bivalent fear of evaluation model of social anxiety: Further integrating findings on fears of positive and negative evaluation. Cognitive Behaviour Therapy, 41, 83-95. doi:10.1080/16506073.2012.661452.

Weeks JW, Heimberg RG and Rodebaugh TL (2008). The Fear of Positive Evaluation Scale: Assessing a proposed cognitive component of social anxiety. Journal of Anxiety Disorders, 22, 44-55. doi:10.1037\%2Fa0025723.

Zou JB and Abbott MJ (2012). Self-perception and rumination in social anxiety. Behaviour Research and Therapy, 50, 250-257. doi:10.1016/j.brat.2012.01.007.

Cite this article: Miers AC (2021). An Investigation Into the Influence of Positive Peer Feedback on Self-Relevant Cognitions in Social Anxiety. Behaviour Change 38, 193-207. https://doi.org/10.1017/bec.2021.8 